\title{
Neurocognitive Effects of Ketamine and Association with Antidepressant Response in Individuals with Treatment-Resistant Depression: A Randomized Controlled Trial
}

\author{
James W Murrough*, 1,2,3,10, Katherine E Burdick 2,3,4,10, Cara F Levitch', Andrew M Perez ${ }^{5}$, Jess W Brallier ${ }^{5}$, \\ Lee C Chang ${ }^{6}$, Alexandra Foulkes ${ }^{7}$, Dennis S Charney ${ }^{1,2,8}$, Sanjay J Mathew ${ }^{9,7}$ and Dan V losifescu ${ }^{1,2,3}$ \\ 'Mood and Anxiety Disorders Program, Department of Psychiatry, Icahn School of Medicine at Mount Sinai, New York, NY, USA; ${ }^{2}$ Fishberg \\ Department of Neuroscience, Icahn School of Medicine at Mount Sinai, New York, NY, USA; ${ }^{3}$ Friedman Brain Institute, Icahn School of Medicine \\ at Mount Sinai, New York, NY, USA; ${ }^{4}$ Department of Psychiatry, Icahn School of Medicine at Mount Sinai, New York, NY, USA; ${ }^{5}$ Department of \\ Anesthesiology, Icahn School of Medicine at Mount Sinai, New York, NY, USA; ${ }^{6}$ Department of Anesthesiology, Baylor College of Medicine, \\ Houston, TX, USA; ${ }^{7}$ Menninger Department of Psychiatry and Behavioral Sciences, Baylor College of Medicine, Houston, TX, USA; ${ }^{8}$ Department \\ of Pharmacology and Systems Therapeutics, Icahn School of Medicine at Mount Sinai, New York, NY, USA; ${ }^{9}$ Michael E. Debakey VA Medical \\ Center, Houston, TX, USA
}

\begin{abstract}
The glutamate N-methyl-D-aspartate (NMDA) receptor antagonist ketamine displays rapid antidepressant effects in patients with treatment-resistant depression (TRD); however, the potential for adverse neurocognitive effects in this population has not received adequate study. The current study was designed to investigate the delayed neurocognitive impact of ketamine in TRD and examine baseline antidepressant response predictors in the context of a randomized controlled trial. In the current study, 62 patients (mean age $=46.2 \pm$ 12.2) with TRD free of concomitant antidepressant medication underwent neurocognitive assessments using components of the MATRICS Consensus Cognitive Battery (MCCB) before and after a single intravenous infusion of ketamine $(0.5 \mathrm{mg} / \mathrm{kg})$ or midazolam $(0.045 \mathrm{mg} / \mathrm{kg})$. Participants were randomized to ketamine or midazolam in a 2:I fashion under double-blind conditions and underwent depression symptom assessments at 24, 48, $72 \mathrm{~h}$, and 7 days post treatment using the Montgomery-Asberg Depression Rating Scale (MADRS). Post-treatment neurocognitive assessment was conducted once at 7 days. Neurocognitive performance improved following the treatment regardless of treatment condition. There was no differential effect of treatment on neurocognitive performance and no association with antidepressant response. Slower processing speed at baseline uniquely predicted greater improvement in depression at $24 \mathrm{~h}$ following ketamine $(t=2.3, p=0.027)$, while controlling for age, depression severity, and performance on other neurocognitive domains. In the current study, we found that ketamine was devoid of adverse neurocognitive effects at 7 days post treatment and that slower baseline processing speed was associated with greater antidepressant response. Future studies are required to further define the neurocognitive profile of ketamine in clinical samples and to identify clinically useful response moderators.
\end{abstract}

Neuropsychopharmacology (2015) 40, 1084-1090; doi:I0.1038/npp.2014.298; published online 17 December 20I4

\section{INTRODUCTION}

Ketamine is a high-affinity, noncompetitive $N$-methyl-Daspartate (NMDA) glutamate receptor antagonist that has demonstrated rapid antidepressant effects in patients with treatment-resistant depression (TRD; Mathew et al, 2010; Murrough et al, 2013a; Murrough et al, 2013b; Zarate et al, 2006). We recently reported that ketamine was superior to an anesthetic control condition (the benzodiazepine midazolam)

* Correspondence: Dr JW Murrough, Mood and Anxiety Disorders Program, Department of Psychiatry, Icahn School of Medicine at Mount Sinai, One Gustave L. Levy Place, Box 1230, New York, NY 10029, USA, Tel: + I 212 24| 7574, Fax: + I 212 24| 3354,

E-mail: james.murrough@mssm.edu

${ }^{10}$ These authors contributed equally to this work.

Received 27 July 20 |4; revised 13 October 20 I4; accepted 18 October 20।4; accepted article preview online 6 November 2014 at rapidly reducing depressive symptoms $24 \mathrm{~h}$ following a single intravenous (IV) infusion in a two-site randomized controlled trial (response rates to ketamine and midazolam were 64 and 28\%, respectively; Murrough et al, 2013a). Randomized controlled trials have also found positive therapeutic effects of ketamine in bipolar depression (Zarate et al, 2012) and posttraumatic stress disorder (PTSD; Feder et al, 2014). Despite the potential of ketamine as a mechanistically novel therapeutic option for patients with TRD, important concerns regarding safety and toxicity remain (Green and Cote, 2009; Morgan et al, 2009). There is a paucity of data on the neurocognitive effects of ketamine in mood disorder populations, thereby hampering risk-benefit analyses used to inform the potential development of ketamine as a treatment for severe or refractory mood disorders.

At the sub-anesthetic doses utilized in depression studies (most commonly $0.5 \mathrm{mg} / \mathrm{kg}$ administered as a slow 40 -min 
infusion), ketamine results in acute dissociative and cognitive effects that typically peak immediately following drug administration and resolve within minutes or up to $2 \mathrm{~h}$ following cessation of the drug (Mathew et al, 2010; Murrough et al, 2013a; Murrough et al, 2013c; Zarate et al, 2006). In healthy volunteers, ketamine reliably results in acute impairments in learning and memory (Krystal et al, 1994; Krystal et al, 1999; Krystal et al, 2005; Morgan et al, 2004; Parwani et al, 2005; Perry et al, 2007). Most studies of neurocognition in healthy volunteers have utilized a dosing strategy distinct from what has been utilized in clinical trials for depression to date. For example, Krystal et al (2005) used a 1-min IV infusion of $0.23 \mathrm{mg} / \mathrm{kg}$ of ketamine followed by a 1 -h infusion of $0.5 \mathrm{mg} / \mathrm{kg}$ in a neurocognitive study in healthy volunteers and assessments were conducted during the drug infusion. Some studies have found evidence for selective impairments in aspects of executive functioning related to ketamine (Krystal et al, 1994; Krystal et al, 1999), whereas other studies have found no impairments (Morgan et al, 2004; Parwani et al, 2005).

We previously described the acute neurocognitive effects of ketamine $(0.5 \mathrm{mg} / \mathrm{kg})$ and the relationship between neurocognition and treatment response in 26 individuals with TRD in the context of a single infusion, open-label clinical trial (Mathew et al, 2010; Murrough et al, 2013c). Using the MATRICS Consensus Cognitive Battery (MCCB) (Nuechterlein et al, 2008) to quantify cognitive functioning at baseline and repeating a subset of tests at $40 \mathrm{~min}$ post treatment, we found that ketamine was associated with select impairments in memory recall (Murrough et al, 2013c), consistent with prior reports in healthy individuals (Krystal et al, 1994; Krystal et al, 1999; Krystal et al, 2005). Further, we found that baseline cognitive functioning was associated with subsequent antidepressant response to ketamine at $24 \mathrm{~h}$ (Murrough et al, 2013c). Individuals who demonstrated a positive antidepressant response to ketamine had slower processing speed at baseline, compared with individuals who did not respond. These preliminary observations suggest a role for dopamine neurotransmission and neural circuits involving the striatum and interconnected regions of prefrontal cortex in the antidepressant mechanism of action of ketamine (Cervenka et al, 2008; Cropley et al, 2006).

The current study was designed to replicate and extend our previous findings regarding ketamine and neurocognition in the context of a two-site, double-blind, randomized controlled trial in TRD patients. The aims were to (1) characterize the effect of ketamine on neurocognitive performance 7 days post treatment, and to (2) investigate baseline neurocognitive predictors of the antidepressant response to ketamine. We hypothesized that 7 days after a single ketamine infusion, there would be no worsening of neurocognitive functioning and that greater depression symptom improvement following ketamine would be associated with slower processing speed at baseline.

\section{MATERIALS AND METHODS}

This neurocognitive study took place during a randomized controlled trial of ketamine in individuals with TRD conducted at two academic medical centers (Baylor College of Medicine and Icahn School of Medicine at Mount Sinai) between November 2010 and August 2012. The primary clinical trial outcomes were previously reported by Murrough et al (2013a) (ClinicalTrials.gov identifier NCT00768430). The institutional review boards at both sites approved the study and participants provided written informed consent before study participation.

\section{Participants}

We enrolled individuals 21-80 years of age, with a primary diagnosis of major depressive disorder (MDD) as assessed with the Structured Clinical Interview for DSM-IV-Patient Edition (SCID; First et al, 1995), and with an inadequate response to at least three therapeutic trials of an antidepressant according to the criteria of the Antidepressant Treatment History Form (Sackeim, 2001). Additional inclusion criteria were a history of at least one previous major depressive episode before the current episode (recurrent MDD) or a chronic major depressive episode (at least 2 years' duration) and a score of 32 or greater on the Inventory of Depressive Symptomatology-Clinician Rated at screening and $24 \mathrm{~h}$ before randomization (IDS- $\mathrm{C}_{30}$; Rush et al, 1996). Patients were excluded if they had a lifetime history of a psychotic illness or bipolar disorder, alcohol or substance abuse in the previous 2 years, unstable medical illness, serious and imminent suicidal or homicidal risk, a score $<27$ on the Mini-Mental State Examination (Folstein et al, 1975), or if they were taking contraindicated medications. For participants taking antidepressant medication, a washout period of at least 1 week was required before enrollment (4 weeks for fluoxetine). Each patient had a physical examination, routine hematologic and biochemical tests, urine toxicology, and an electrocardiogram to detect unstable medical illness or substance use.

\section{Study Design}

Study participants were free of concomitant antidepressants and other psychotropic medications for the duration of the study with the exception of a stable dose of a nonbenzodiazepine hypnotic. Eligible participants underwent a battery of neurocognitive tests (see below for details) within 1 week before receiving a single IV infusion of ketamine $(0.5 \mathrm{mg} / \mathrm{kg})$ or midazolam $(0.045 \mathrm{mg} / \mathrm{kg})$ over $40 \mathrm{~min}$ under double-blind conditions in an inpatient clinical research setting. Participants were allocated to the ketamine or midazolam in a 2:1 ratio. Midazolam is a benzodiazepine anesthetic agent used as an 'active placebo' in the current study to control for non-specific treatment effects related to sedation or other acute effects of anesthetic drug administration. Detailed study drug infusion methods were presented previously (Murrough et al, 2013a). A trained rater conducted symptom ratings at fixed intervals during the infusion and for $240 \mathrm{~min}$ following the start of the infusion. Patients were discharged from the research unit $24 \mathrm{~h}$ following the infusion, and received outpatient evaluations 48 and $72 \mathrm{~h}$ and 7 days post infusion. The neurocognitive battery was repeated once at 7 days post treatment using alternative test forms to minimize practice effects. Neuropsychology raters were masked to drug identity and day-ofinfusion side effects. 
Change in depression severity was measured using the Montgomery-Asberg Depression Rating Scale (MADRS; Montgomery and Asberg, 1979); categorical response was defined as $\geqslant 50 \%$ reduction in MADRS score relative to baseline. The primary depression outcome for the clinical trial was MADRS score $24 \mathrm{~h}$ following treatment. Secondary outcomes included MADRS score at 48 and $72 \mathrm{~h}$ and 7 days following treatment and the proportion of responders at each of the aforementioned time points. See Figure 1 for an overview of the study design.

\section{Neurocognitive Assessment}

Neurocognitive functioning was assessed at baseline and at 7 days post treatment using a subset of the MCCB (Trails A, Wechsler Memory Scale (WMS) Spatial Span, Brief Assessment of Cognition in Schizophrenia (BACS) Digit Symbol, Letter-Number Sequencing, Hopkins Verbal Learning Test (HVLT), Brief Visual Memory Test (BVMT), Neuropsychological Assessment Battery (NAB) Mazes, and Category Fluency). We computed standardized scores ( $T$-scores with a mean of 50 and an SD of 10) for the MCCB data using the MCCB computerized scoring program, which includes correction for age and sex based on the MCCB normative sample.

To compute neurocognitive domain scores, we calculated mean $T$-scores as follows: processing speed (category fluency, Trails A, BACS Digit Symbol); working memory (WMS-III Spatial Span, letter-number); verbal learning (HVLT learning and delay); visual learning (BVMT learning); and reasoning/problem solving (NAB Mazes).

\section{Statistical Analysis}

Characteristics of the study sample are described using summary statistics. Univariate tests were used to compare the ketamine and midazolam treatment groups on baseline characteristics. The effect of time (baseline vs 7 days), treatment condition (ketamine vs midazolam), and antidepressant response status (responder vs non-responder) on cognitive performance and interaction effects were evaluated using a set of repeated measures analysis of variance (ANOVA) models. All models included change in

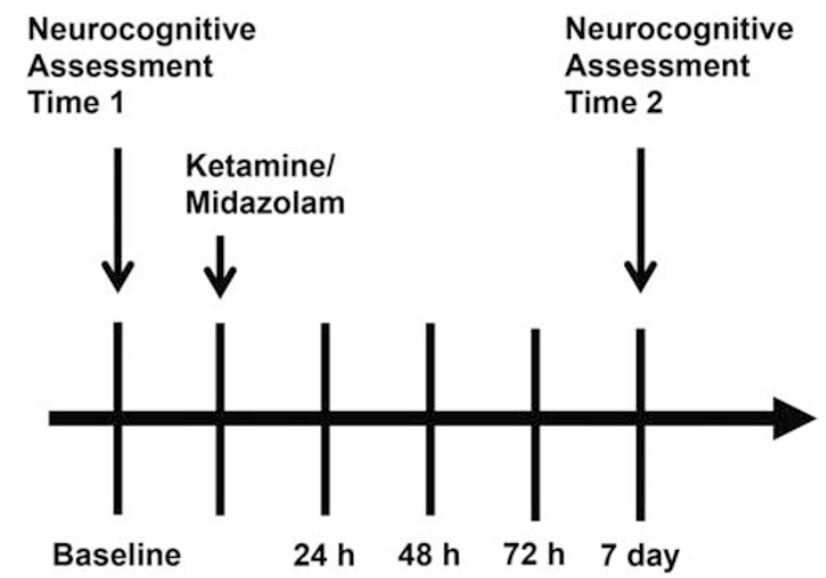

Figure I Study design. Figure depicts timing of neurocognitive assessments relative to the treatment intervention with ketamine or midazolam. Depression assessments were conducted at all study visits. depression severity from baseline to 7 days as a co-variate to evaluate and control for the potential effects of improved depression on cognition. We considered the influence of responder status at both $24 \mathrm{~h}$ (the time point corresponding to the primary clinical trial outcome) and 7 days (the time point corresponding to the post-treatment neurocognitive assessment) on neurocognitive performance in separate sets of models.

To evaluate the influence of baseline cognitive performance on antidepressant response, we entered the five neurocognitive domain scores, baseline symptom ratings, demographics, and a calculated depressive symptom change score (MADRS $24 \mathrm{~h}$ minus MADRS Baseline) into a backwards stepwise linear regression to identify clinical and cognitive predictors of ketamine response. To follow up on significant results, we compared outcome groups (responder vs non-responder) using ANOVA and logistic regression.

\section{RESULTS}

\section{Demographic and Clinical Characteristics}

Seventy-three individuals were randomized and $n=72$ received study medication and constituted the modified intention to treat sample ( $n=47$ received ketamine, $n=25$ received midazolam) in the parent clinical trial (Murrough et al, 2013a). Data were missing or incomplete for four patients in the ketamine group and six patients in the midazolam group. Therefore, the current neurocognitive sub-study included 43 individuals randomized to ketamine and 19 individuals randomized to midazolam. One domain (visual learning) had additional missing data with a sample size of $n=34$ for ketamine and $n=17$ for midazolam. Baseline sample characteristics are presented in Table 1. Treatment groups had similar ages, gender frequencies, depression symptom severity, and level of treatment resistance.

\section{Effect of Time, Treatment Condition, and Antidepressant Response on Cognitive Performance}

At the 7-day post-treatment assessment, we found a significant main effect of time such that, on average, participants' performance improved from baseline in the cognitive domains of processing speed $\left(\mathrm{F}_{1,59}=6.58, p=0.013\right)$, verbal learning $\left(\mathrm{F}_{1,59}=6.80, p=0.012\right)$, and visual learning $\left(\mathrm{F}_{1,48}=6.48, p=0.014\right)$ across both treatment conditions while controlling for change in depression symptoms over the same time interval. There was no change in the domains of working memory or reasoning. There was no specific effect of ketamine on cognitive performance (no main effect of treatment condition), and no effect of antidepressant response measured either at $24 \mathrm{~h}$ or 7 days on cognitive performance. There were no significant interaction effects (Figure 2).

\section{Baseline Cognitive Functioning and Antidepressant Response to Ketamine}

We evaluated the influence of baseline cognitive performance on change in MADRS score from baseline to $24 \mathrm{~h}$ 
Table I Characteristics of Study Sample

\begin{tabular}{|c|c|c|c|c|c|}
\hline Characteristic & Total sample $(n=62)$ & Ketamine $(n=43)$ & Midazolam $(n=19)$ & Statistic & $P$-value \\
\hline Age (years) & $46.1 \pm 12.2$ & $47.1 \pm 12.6$ & $43.8 \pm 11.0$ & $t=0.98$ & 0.33 \\
\hline Female & $34(54.8 \%)$ & $24(57.0 \%)$ & $10(50.0 \%)$ & $\chi^{2}=0.82$ & 1.0 \\
\hline Age at first major depressive episode (years) & $22.8 \pm 11.4$ & $22.4 \pm 11.0$ & $23.9 \pm 12.5$ & $t=0.49$ & 0.62 \\
\hline Duration of illness (years) & $23.1 \pm 13.3$ & $24.6 \pm 12.8$ & $19.8 \pm 14.2$ & $t=1.3$ & 0.20 \\
\hline Duration of current episode (months) & $138 \pm 156$ & $150 \pm 163$ & $110 \pm 143$ & $t=0.93$ & 0.36 \\
\hline
\end{tabular}

Values indicate mean \pm SD or count (\%). MADRS, Montgomery-Asberg Depression Rating Scale; MADRS scores range from 0 to 60 , with higher scores indicating more severe symptoms. Univariate statistical tests compare values between ketamine and midazolam treatment conditions using independent sample $t$-test or chisquare as appropriate.

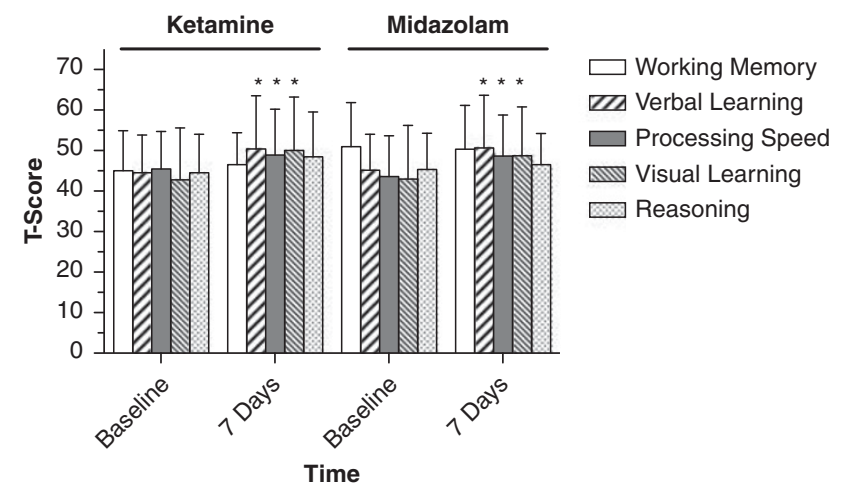

Figure 2 Neurocognitive performance over time in ketamine and midazolam treatment groups in a randomized controlled trial in treatmentresistant major depression. Figure depicts mean $( \pm \mathrm{SD})$ T-scores in patients with treatment-resistant depression using a subset of cognitive domains derived from the MATRICS Consensus Cognitive Battery. Cognitive measurements were performed at baseline and 7 days following a single intravenous infusion of ketamine $(0.5 \mathrm{mg} / \mathrm{kg})$ or midazolam $(0.045 \mathrm{mg} / \mathrm{kg})$. *Indicates significant improvement in T-score over time regardless of treatment condition $(p<0.05)$. There was no significant main effect of treatment and no significant treatment $\times$ time interaction.

using backwards stepwise linear regression with the following predictor variables in the model: age, baseline MADRS score, processing speed, working memory, verbal learning, visual learning, and reasoning. The final and most significant model contained the single predictor variable of processing speed $(\mathrm{F}(1,37)=5.3, p=0.027$; beta $=0.43 \pm$ $0.19, t=2.3, p=0.027$ ), wherein poor processing speed at baseline was associated with improved antidepressant response to ketamine. Using the categorical definition of response as the outcome, we conducted a backwards stepwise logistic regression and included the same predictor variables as in the prior linear regression. The most significant model contained processing speed and visual learning $\left(\chi^{2}=11.82, p=0.003\right)$; only processing speed was significantly associated with the response variable (Exp (beta) $=0.823$, Wald $=7.76, p=0.005$ ). Follow up, univariate tests comparing responders to non-responders on each neurocognitive domain confirmed that ketamine responders had significantly slower processing speed at baseline $(T$-score $=43.37 \pm 8.78)$ compared with ketamine non-responders $\left(T\right.$-score $\left.=49.24 \pm 10.1 ; \mathrm{F}_{1,45}=4.36, p=0.043\right)$. There were no differences between the response groups on other cognitive measures. We found no evidence of association between baseline processing speed and baseline depression severity $(r=-0.167, p=0.164)$.

We found no significant relationship between baseline cognitive performance and antidepressant response to midazolam (data not shown).

\section{DISCUSSION}

In a randomized controlled trial in patients with TRD, we found that a single sub-anesthetic dose of ketamine had no deleterious effect on neurocognitive performance 7 days following treatment compared with midazolam. Performance on the measures of processing speed, verbal learning, and visual learning improved at study end compared with baseline regardless of treatment condition or change in depression severity, likely reflecting a non-specific learning effect. Finally, we replicated our previous finding (Murrough et al, 2013c) that slower baseline processing speed predicted a rapid antidepressant response at $24 \mathrm{~h}$ following ketamine.

Ketamine is associated with acute perceptual and cognitive disturbances at the time of drug administration (Krystal et al, 2005; Morgan et al, 2004), and chronic ketamine abuse can lead to persistent neurocognitive impairments (Morgan and Curran, 2006; Morgan et al, 2009) and potentially deleterious brain changes measured using in vivo neuroimaging (Edward Roberts et al, 2014). The impact of ketamine on neurocognitive function in patients with TRD, however, has received only minimal study to date. Our group first reported circumscribed memory impairment immediately following a single ketamine dose $(0.5 \mathrm{mg} / \mathrm{kg})$ administered as a slow infusion over $40 \mathrm{~min}$ (Murrough et al, 2013c). Subsequently, two open-label studies explored the neurocognitive effects of up to six ketamine infusions in patients with treatment-resistant unipolar or bipolar depression and found no evidence of impairment (Diamond et al, 2014; Shiroma et al, 2014). The current study featured a relatively large sample size and a two-site, randomized controlled design and yielded results consistent with these prior reports. 
Our finding of an absence of adverse effects of ketamine on neurocognitive functioning in this patient population may contribute to a risk-benefit analysis of ketamine utilization as a treatment for refractory depression. The current gold standard treatment for refractory depression-electroconvulsive therapy (ECT) - may provide an appropriate safety comparison for ketamine. Cognitive impairment is the most significant side effect limiting the use of ECT, although the extent of short- and longer-term adverse cognitive effects continues to be debated (Semkovska and McLoughlin, 2010). Our finding of an absence of adverse cognitive effects of ketamine 7 days post treatment suggests that ketamine may compare favorably to ECT. The present study examined only the impact of a single ketamine treatment on delayed performance and did not measure the immediate effects of ketamine on neurocognition, nor the effects of repeated ketamine treatments. If ketamine were to be approved for use in clinical practice, it would very likely be administered in a repeated fashion over weeks, months, or longer. Although very early data on repeated infusions exist (Diamond et al, 2014; Shiroma et al, 2014), much more research will be required to establish the potential cognitive risks of longer-term repeated ketamine treatments for patients with severe or refractory forms of mood disorders.

In the current study, we replicated our previous finding that slow baseline processing speed is associated with improved symptom reduction in TRD following ketamine (Murrough et al, 2013c). Notably, processing speed was not associated with depression symptom severity at baseline. Our finding is also broadly consistent with a recent report describing an association between reduced attention at baseline and improved antidepressant response to ketamine (Shiroma et al, 2014). Both processing speed and attention have been linked to dopamine functioning within prefrontal-subcortical circuits (Cropley et al, 2006), and ketamine is known to modulate dopamine signaling within the striatum and prefrontal cortex in animals (Moghaddam et al, 1997) and humans (Kegeles et al, 2000; Rabiner, 2007; Smith et al, 1998). Mechanistic studies in animals show that ketamine rapidly enhances synaptic plasticity at the level of prefrontal cortical neurons ( $\mathrm{Li}$ et al, 2010, 2011), and is able to rapidly reverse stress-induced dendritic atrophy and behavioral depression in a brain-derived neurotrophic factor (BDNF)-dependent manner (Li et al, 2011; Liu et al, 2012). A recent study found that ketamine reversed deficit dopamine signaling in a learned helplessness model of depression and normalized synaptic plasticity within the nucleus accumbens (indexed by long-term potentiation) via activation of dopamine D1 receptors (Belujon and Grace, 2014). Chronic and/or uncontrollable forms of stress are known to reduce dopamine signaling at the level of the ventral tegmental area (VTA) and striatum (Cabib and Puglisi-Allegra, 2012), and these data suggest that ketamine may alleviate depressive symptoms, at least in part, via modulation of dopamine signaling. A recent study utilizing [18F]-fluorodeoxyglucose (FDG) positron emission tomography (PET) in patients with bipolar depression found that improvement in depression score was associated with increased metabolism within the ventral striatum (Nugent et al, 2014). No study to date has directly examined the relationship between dopamine signaling and antide- pressant response to ketamine, and the role of dopamine in the mechanism of action of ketamine remains unknown. Further study in patient populations with the use of in vivo imaging will be required to define the antidepressant mechanisms of ketamine in humans.

Our results provide early support for the development of a neurocognitive pre-treatment predictor of response to ketamine in depressed patients. Prior studies have found associations between clinical and demographic factors and therapeutic response to conventional antidepressants (Perlis, 2013), however, a quantitative laboratory-based behavioral or biological predictor of treatment response has remained elusive (Kapur et al, 2012; Simon and Perlis, 2010; Trivedi, 2013). Prior research involving ketamine for unipolar or bipolar depression has suggested candidate clinical or demographic variables associated with therapeutic response, including a family history of alcoholism and a higher body mass index (BMI) (Niciu et al, 2014). Candidate baseline biological predictors of antidepressant response to ketamine previously reported include a single-nucleotide polymorphism in the gene coding for BDNF (Laje et al, 2012), as well as differential responses to emotional faces within the anterior cingulate cortex during magnetoencephalography (Salvadore et al, 2009). It may be that no one single biobehavioral marker will provide a sufficient level of precision and discrimination so as to provide a clinically useful tool for personalizing ketamine treatment in depression (Zarate et al, 2013). Our study suggests that specific neurocognitive measurements may hold promise as a component of a 'bio-signature,' guiding personalized treatment for patients who are candidates for ketamine therapy in the future.

Our study has several limitations. A total of 62 patients with TRD underwent neurocognitive evaluation before and after ketamine treatment across two academic medical centers. Although the sample size of the current study is comparatively large, it is likely that significantly larger studies will be required to define clinically meaningful biological or neurocognitive markers of treatment response. It will be important going forward for clinical studies of ketamine or other mechanistically novel rapid-acting antidepressants to utilize standardized neurocognitive assessments to avoid the problem of 'approximate replication' (Kapur et al, 2012). For example, the study by Shiroma et al (2014) found that impaired attention, but not impaired processing speed per se, was associated with improved antidepressant response to ketamine. This discrepancy could be, in part, due to the differences in neurocognitive assessments utilized (the MCCB was utilized in the current study, compared with the CogState battery (Collie et al, 2003) in the study by Shiroma et al (2014)). The current study examined neurocognitive function using a comprehensive battery, but optimal biomarker assessments will likely require multi-modal data acquisition, potentially including neuroimaging (Zarate et al, 2013), quantitative electroencephalography (qEEG) (Cook et al, 2013), and peripheral blood markers (Uddin, 2014). The current study did not include a specific measure of attention and, therefore, we are unable to directly compare our findings with those of Shiroma et al (2014). Regarding safety, the current study examined the effects of a single dose of ketamine administered at one time point. While this dose 
has been previously found to result in rapid onset therapeutic effects (Mathew et al, 2010; Murrough et al, 2013a; Murrough et al, 2013b; Zarate et al, 2006), future studies will be required to explore the wide range of potential dose and treatment frequency parameters to optimize the treatment for individual patients. In the current study, we did not measure the cognitive effects of ketamine immediately post administration (eg, at $40 \mathrm{~min}$ from the start of the 40-min infusion), as we had done in our previous study (Murrough et al, 2013c). Therefore, we were unable to assess the relationship between immediate cognitive effects and antidepressant outcome. The potential for ketamine to result in adverse neurocognitive effects over a longer time frame in the context of repeated treatment will require careful study.

In conclusion, we report herein that ketamine is devoid of short-term adverse neurocognitive effects in patients with TRD at the studied dose and that reduced processing speed at baseline is associated with a more robust antidepressant response. Future studies will be required to define the optimal treatment parameters and identify clinically useful response moderators for ketamine as a treatment for refractory forms of mood disorders.

\section{FUNDING AND DISCLOSURE}

This work was supported by National Institutes of Health (NIH)/National Institute of Mental Health (NIMH) grant R01MH081870 (SJM), UL1TR000067 from the NIH National Center for Advancing Translational Sciences (Mount Sinai CTSA), Department of Veterans Affairs, and a NARSAD Independent Investigator Award (SJM). This work was supported with resources and the use of facilities at the Michael E. DeBakey VA Medical Center, Houston, TX. Dr Mathew is supported in part by the Marjorie Bintliff Johnson and Raleigh White Johnson, Jr Chair for Research in Psychiatry at Baylor College of Medicine. Dr Murrough is supported by a Career Development Award from NIH/ NIMH (K23MH094707). The content is solely the responsibility of the authors and does not necessarily represent the official views of the NIH or other funding agency.

In the past 3 years, Dr Murrough has served on advisory boards for Janssen Research and Development and Genentech, has provided consultation services for ProPhase, LLC and Impel Neuropharma, and has received research support from Janssen and Avanir Pharmaceuticals; he is named on a patent pending for neuropeptide $\mathrm{Y}$ as a treatment for mood and anxiety disorders. Dr Burdick has served on advisory boards for Dainippon Sumitomo Pharma and Takeda/ Lundbeck. Dr Iosifescu has consulted for Avanir, CNS Response, INSYS Therapeutics, Lundbeck, Otsuka, Servier, Sunovion and he has received grant/research support through Mount Sinai School of Medicine from Alkermes, Astra Zeneca, Brainsway, Euthymics Bioscience Inc, Neosync, Roche and Shire. Dr Dennis Charney (Dean of Icahn School of Medicine at Mount Sinai) and Icahn School of Medicine at Mount Sinai have been named on a use patent on ketamine for the treatment of depression. The Icahn School of Medicine has entered into a licensing agreement for the use of ketamine as therapy for treatment-resistant depression. Dr Charney and Icahn School of Medicine at Mount Sinai could potentially benefit if ketamine were to gain approval for the treatment of depression. Dr Charney is named on a patent pending for ketamine as a treatment for PTSD and for neuropeptide $\mathrm{Y}$ as a treatment for mood and anxiety disorders; he has received funding from the U.S. Department of Defense, NIH, NIH/NIMH, NARSAD, USAMRAA; he has severed on the scientific advisory board for the Institute of Medicine Committee on DHS Workforce Resilience and on the editorial board of CNS Spectrums. Dr Mathew has received consulting fees from Bristol-Myers Squibb, Cerecor, Genentech, and Naurex, and research support from AstraZeneca, Janssen Research and Development, and Otsuka. All other authors declare no conflict of interest.

\section{REFERENCES}

Belujon P, Grace AA (2014). Restoring mood balance in depression: ketamine reverses deficit in dopamine-dependent synaptic plasticity. Biol Psychiatry (e-pub ahead of print).

Cabib S, Puglisi-Allegra S (2012). The mesoaccumbens dopamine in coping with stress. Neurosci Biobehav Rev 36: 79-89.

Cervenka S, Backman L, Cselenyi Z, Halldin C, Farde L (2008). Associations between dopamine D2-receptor binding and cognitive performance indicate functional compartmentalization of the human striatum. Neuroimage 40: 1287-1295.

Collie A, Maruff P, Darby DG, McStephen M (2003). The effects of practice on the cognitive test performance of neurologically normal individuals assessed at brief test-retest intervals. J Int Neuropsychol Soc 9: 419-428.

Cook IA, Hunter AM, Gilmer WS, Iosifescu DV, Zisook S, Burgoyne KS et al (2013). Quantitative electroencephalogram biomarkers for predicting likelihood and speed of achieving sustained remission in major depression: a report from the biomarkers for rapid identification of treatment effectiveness in major depression (BRITE-MD) trial. J Clin Psychiatry 74: 51-56.

Cropley VL, Fujita M, Innis RB, Nathan PJ (2006). Molecular imaging of the dopaminergic system and its association with human cognitive function. Biol Psychiatry 59: 898-907.

Diamond PR, Farmery AD, Atkinson S, Haldar J, Williams N, Cowen PJ et al (2014). Ketamine infusions for treatment resistant depression: a series of 28 patients treated weekly or twice weekly in an ECT clinic. J Psychopharmacol 28: 536-544.

Edward Roberts R, Curran HV, Friston KJ, Morgan CJ (2014). Abnormalities in white matter microstructure associated with chronic ketamine use. Neuropsychopharmacology 39: 329-338.

Feder A, Parides MK, Murrough JW, Perez AM, Morgan JE, Saxena $S$ et al (2014). Efficacy of intravenous ketamine for treatment of chronic posttraumatic stress disorder: a randomized clinical trial. JAMA Psychiatry 71: 681-688.

First MB, Spitzer RL, Gibbon M, Williams JBW (1995). Structured Clinical Interview for DSM-IV Axis Disorders (SCID). New York State Psychiatric Institute, Biometrics Research: New York, NY, USA.

Folstein MF, Folstein SE, McHugh PR (1975). 'Mini-mental state'. A practical method for grading the cognitive state of patients for the clinician. J Psychiatr Res 12: 189-198.

Green SM, Cote CJ (2009). Ketamine and neurotoxicity: clinical perspectives and implications for emergency medicine. Ann Emerg Med 54: 181-190.

Kapur S, Phillips AG, Insel TR (2012). Why has it taken so long for biological psychiatry to develop clinical tests and what to do about it? Mol Psychiatry 17: 1174-1179.

Kegeles LS, Abi-Dargham A, Zea-Ponce Y, Rodenhiser-Hill J, Mann JJ, Van Heertum RL et al (2000). Modulation of amphetamineinduced striatal dopamine release by ketamine in humans: implications for schizophrenia. Biol Psychiatry 48: 627-640. 
Krystal JH, D'Souza DC, Karper LP, Bennett A, Abi-Dargham A, Abi-Saab D et al (1999). Interactive effects of subanesthetic ketamine and haloperidol in healthy humans. Psychopharmacology (Berl) 145: 193-204.

Krystal JH, Karper LP, Seibyl JP, Freeman GK, Delaney R, Bremner JD et al (1994). Subanesthetic effects of the noncompetitive NMDA antagonist, ketamine, in humans. Psychotomimetic, perceptual, cognitive, and neuroendocrine responses. Arch Gen Psychiatry 51: 199-214.

Krystal JH, Perry EB Jr, Gueorguieva R, Belger A, Madonick SH, Abi-Dargham A et al (2005). Comparative and interactive human psychopharmacologic effects of ketamine and amphetamine: implications for glutamatergic and dopaminergic model psychoses and cognitive function. Arch Gen Psychiatry 62: 985-994.

Laje G, Lally N, Mathews D, Brutsche N, Chemerinski A, Akula N et al (2012). Brain-derived neurotrophic factor Val66Met polymorphism and antidepressant efficacy of ketamine in depressed patients. Biol Psychiatry 72: e27-e28.

Li N, Lee B, Liu RJ, Banasr M, Dwyer JM, Iwata M et al (2010). mTOR-dependent synapse formation underlies the rapid antidepressant effects of NMDA antagonists. Science 329: 959-964.

Li N, Liu RJ, Dwyer JM, Banasr M, Lee B, Son H et al (2011). Glutamate $N$-methyl-D-aspartate receptor antagonists rapidly reverse behavioral and synaptic deficits caused by chronic stress exposure. Biol Psychiatry 69: 754-761.

Liu RJ, Lee FS, Li XY, Bambico F, Duman RS, Aghajanian GK (2012). Brain-derived neurotrophic factor Val66Met allele impairs basal and ketamine-stimulated synaptogenesis in prefrontal cortex. Biol Psychiatry 71: 996-1005.

Mathew SJ, Murrough JW, aan het Rot M, Collins KA, Reich DL, Charney DS (2010). Riluzole for relapse prevention following intravenous ketamine in treatment-resistant depression: a pilot randomized, placebo-controlled continuation trial. Int J Neuropsychopharmacol 13: 71-82.

Moghaddam B, Adams B, Verma A, Daly D (1997). Activation of glutamatergic neurotransmission by ketamine: a novel step in the pathway from NMDA receptor blockade to dopaminergic and cognitive disruptions associated with the prefrontal cortex. J Neurosci 17: 2921-2927.

Montgomery SA, Asberg M (1979). A new depression scale designed to be sensitive to change. Br J Psychiatry 134: 382-389.

Morgan CJ, Curran HV (2006). Acute and chronic effects of ketamine upon human memory: a review. Psychopharmacology (Berl) 188: 408-424.

Morgan CJ, Mofeez A, Brandner B, Bromley L, Curran HV (2004). Acute effects of ketamine on memory systems and psychotic symptoms in healthy volunteers. Neuropsychopharmacology 29: 208-218.

Morgan CJ, Muetzelfeldt L, Curran HV (2009). Ketamine use, cognition and psychological wellbeing: a comparison of frequent, infrequent and ex-users with polydrug and non-using controls. Addiction 104: 77-87.

Murrough JW, Iosifescu DV, Chang LC, Al Jurdi RK, Green CE, Perez AM et al (2013a). Antidepressant efficacy of ketamine in treatment-resistant major depression: a two-site randomized controlled trial. Am J Psychiatry 170: 1134-1142.

Murrough JW, Perez AM, Pillemer S, Stern J, Parides MK, aan het Rot $M$ et al (2013b). Rapid and longer-term antidepressant effects of repeated ketamine infusions in treatment-resistant major depression. Biol Psychiatry 74: 250-256.

Murrough JW, Wan LB, Iacoviello B, Collins KA, Solon C, Glicksberg B et al (2014). Neurocognitive effects of ketamine in treatment-resistant major depression: association with antidepressant response. Psychopharmacology 231: 481.

Niciu MJ, Luckenbaugh DA, Ionescu DF, Guevara S, Machado-Vieira R, Richards EM et al (2014). Clinical predictors of ketamine response in treatment-resistant major depression. J Clin Psychiatry 75: e417-e423.

Nuechterlein KH, Green MF, Kern RS, Baade LE, Barch DM, Cohen JD et al (2008). The MATRICS Consensus Cognitive Battery, part 1: test selection, reliability, and validity. Am J Psychiatry 165: 203-213.

Nugent AC, Diazgranados N, Carlson PJ, Ibrahim L, Luckenbaugh DA, Brutsche $\mathrm{N}$ et al (2014). Neural correlates of rapid antidepressant response to ketamine in bipolar disorder. Bipolar Disord 16: 119-128.

Parwani A, Weiler MA, Blaxton TA, Warfel D, Hardin M, Frey K et al (2005). The effects of a subanesthetic dose of ketamine on verbal memory in normal volunteers. Psychopharmacology (Berl) 183: 265-274.

Perlis RH (2013). A clinical risk stratification tool for predicting treatment resistance in major depressive disorder. Biol Psychiatry 74: 7-14.

Perry EB Jr, Cramer JA, Cho HS, Petrakis IL, Karper LP, Genovese A et al Yale Ketamine Study Group (2007). Psychiatric safety of ketamine in psychopharmacology research. Psychopharmacology (Berl) 192: 253-260.

Rabiner EA (2007). Imaging of striatal dopamine release elicited with NMDA antagonists: is there anything there to be seen? J Psychopharmacol 21: 253-258.

Rush AJ, Gullion CM, Basco MR, Jarrett RB, Trivedi MH (1996). The Inventory of Depressive Symptomatology (IDS): psychometric properties. Psychol Med 26: 477-486.

Sackeim HA (2001). The definition and meaning of treatmentresistant depression. J Clin Psychiatry 62(Suppl 16): 10-17.

Salvadore G, Cornwell BR, Colon-Rosario V, Coppola R, Grillon C, Zarate CA Jr et al (2009). Increased anterior cingulate cortical activity in response to fearful faces: a neurophysiological biomarker that predicts rapid antidepressant response to ketamine. Biol Psychiatry 65: 289-295.

Semkovska M, McLoughlin DM (2010). Objective cognitive performance associated with electroconvulsive therapy for depression: a systematic review and meta-analysis. Biol Psychiatry 68: 568-577.

Shiroma PR, Albott CS, Johns B, Thuras P, Wels J, Lim KO (2014). Neurocognitive performance and serial intravenous subanesthetic ketamine in treatment-resistant depression. Int J Neuropsychopharmacol 17: 1805-1813.

Simon GE, Perlis RH (2010). Personalized medicine for depression: can we match patients with treatments? Am J Psychiatry 167: $1445-1455$.

Smith GS, Schloesser R, Brodie JD, Dewey SL, Logan J, Vitkun SA et al (1998). Glutamate modulation of dopamine measured in vivo with positron emission tomography (PET) and 11Craclopride in normal human subjects. Neuropsychopharmacology 18: $18-25$.

Trivedi MH (2013). Modeling predictors, moderators and mediators of treatment outcome and resistance in depression. Biol Psychiatry 74: 2-4.

Uddin M (2014). Blood-based biomarkers in depression: emerging themes in clinical research. Mol Diagn Ther 18: 469-482.

Zarate CA Jr, Brutsche NE, Ibrahim L, Franco-Chaves J, Diazgranados N, Cravchik A et al (2012). Replication of ketamine's antidepressant efficacy in bipolar depression: a randomized controlled add-on trial. Biol Psychiatry 71: 939-946.

Zarate CA Jr, Mathews DC, Furey ML (2013). Human biomarkers of rapid antidepressant effects. Biol Psychiatry 73: 1142-1155.

Zarate CA Jr, Singh JB, Carlson PJ, Brutsche NE, Ameli R, Luckenbaugh DA et al (2006). A randomized trial of an $\mathrm{N}$-methyl-D-aspartate antagonist in treatment-resistant major depression. Arch Gen Psychiatry 63: 856-864. 